\title{
Os desafios da nanotecnologia para a vigilância sanitária de medicamentos
}

\author{
The challenges of nanotechnology \\ for the sanitary vigilance of medication
}

Ariane de Jesus Sousa Batista ${ }^{1}$

Vera Lúcia Edais Pepe

${ }^{1}$ Departamento de

Administração e

Planejamento em Saúde,

Escola Nacional de Saúde

Pública Sergio Arouca,

Fiocruz. R. Leopoldo

Bulhões 1480, Manguinhos.

21.041-210 Rio de Janeiro

RJ Brasil.

ariane.jesus@gmail.com
Abstract The development of effective and safe nanotechnology medication with a high cost-benefit ratio is a strategic imperative for public health in Brazil. The lack of information demands sanitary regulation geared to protecting health and the environment. This study seeks to assess the current stage of development, the regulatory framework and the challenges facing nanotechnology medication in Brazil, emphasizing sanitary surveillance. Document analysis was conducted in national and international regulatory agency sites. Despite the incipient regulation for this type of medication, 7 registered nanotechnology products were found in Brazil, without clear identification on leaflets or packaging regarding their nanotechnology composition, as well as 4 similar products. Risk assessment and regulation of such medication requires specialized personnel and equipment, as well as the participation of society in the formulation and implementation of regulatory policies. The suggestion proposed is that the regulatory framework should follow the precautionary principle, whereby products are registered as new medication with clear information on the labeling and controlled usage, until further results on the assessment of risk are obtained.

Key words Nanotechnology, Nanotechnology medication, Innovation in health, Sanitary regulation, Sanitary surveillance
Resumo No Brasil, o desenvolvimento de medicamentos nanotecnológicos eficazes, seguros e com alta relação custo/benefício é estratégico para a Saúde Coletiva. A falta de informações requer uma regulação sanitária voltada à proteção da saúde e do ambiente. Objetiva-se identificar o estágio de desenvolvimento e o marco regulatório dos medicamentos nanotecnológicos e seus desafios no Brasil, enfatizando a segurança sanitária. Foi realizada uma análise documental em sítios de agências reguladoras, nacional e internacionais. Apesar da incipiente regulação destes medicamentos, foram encontrados 7 registrados, no Brasil, sem identificação clara na bula e na embalagem quanto às suas constituições nanotecnológicas, e 4 como medicamentos similares. A avaliação de risco e a regulação desses medicamentos exigem pessoas e equipamentos especializados, bem como a participação da sociedade na definição e implementação das politicas regulatórias. Sugere-se que a regulação considere o princípio da precaução, sendo o registro feito como medicamento novo e com informação na rotulagem e uso monitorado, de forma que sejam obtidos novos resultados sobre a avaliação de seu risco.

Palavras-chave Nanotecnologia, Medicamentos nanotecnológicos, Inovação em saúde, Regulação sanitária, Vigilância sanitária 


\section{Introdução}

A nanotecnologia consiste na utilização de materiais ou processos em que ao menos um de seus componentes possua uma de suas dimensões na escala nanométrica ${ }^{1}$. A princípio, qualquer material pode ser reduzido ao tamanho nanométrico $\mathrm{e}$ essa nova tecnologia pode ser aplicada, de forma transdisciplinar, em qualquer campo de atuação, podendo influenciar toda a economia mundial ${ }^{2}$.

Há acelerado crescimento e desenvolvimento dessa tecnologia e, atualmente, podem ser encontrados no mercado diversos produtos nanotecnológicos. A expectativa é que em 2015 o mercado da nanotecnologia atinja US\$ 3,5 trilhões ${ }^{3}$.

Uma das áreas de crescente desenvolvimento da nanotecnologia é a relacionada aos medicamentos, mais especificamente no desenvolvimento de novos sistemas de liberação de fármacos, tendo em vista as vantagens que apresentam em relação à liberação convencional e à projeção de seu crescimento. Acredita-se que, em 2015, os produtos terapêuticos nanotecnológicos representarão um mercado de US\$ 3,4 bilhões ${ }^{4}$.

O Brasil tem interesse estratégico na nanotecnologia e muito já se tem investido em seu desenvolvimento. Na contramão dos países centrais, entretanto, pouco avançou na apropriada regulação dos medicamentos nanotecnológicos que já começam a entrar no mercado ${ }^{4}$. Foladori et al. ${ }^{5}$ identificaram duas características no desenvolvimento da nanotecnologia na América Latina: a busca de melhor competitividade internacional com a criação de centros de excelência científica integrados com a indústria e a falta de atenção no que diz respeito à questão social, tais como a proteção de consumidores e trabalhadores frente a seus riscos potenciais e a não participação da sociedade nas decisões de política pública. Para os autores, não fazem parte das agendas da nanotecnologia na América Latina, mais especificamente México, Argentina e Brasil, três temas chaves de um desenvolvimento voltado à promoção da equidade e redução da pobreza, quais sejam: a participação dos movimentos sociais, a qualificação da força de trabalho e a confiança do consumidor. A participação de movimentos sociais na definição da Política de Ciência e Tecnologia a ser adotada neste âmbito, para além do Estado, empresários e pesquisadores, é importante para incorporar objetivos que estimulem o desenvolvimento de áreas com impacto mais direto no nível de vida da população. No que diz respeito à área farmacêutica, os profissi- onais devem conhecer como se faz a correta manipulação, quais os procedimentos de segurança e qual a infraestrutura adequada, de forma a evitar riscos à saúde e ao ambiente. $\mathrm{O}$ risco à saúde deve ser abordado igualmente no que diz respeito aos consumidores, já que apesar do conhecimento científico produzido, desde a década de noventa, sobre os riscos da nanotecnologia para a saúde humana e para o ambiente ter crescido, ainda permanece insuficiente ${ }^{5}$.

No Brasil, as ações de regulação sanitária, visando à redução da nocividade ou risco para a saúde da população e do meio ambiente são atribuições do Sistema Nacional de Vigilância Sanitária (Visa), que tem o papel de regular a entrada deste tipo de medicamento no mercado, assim como fiscalizar e monitorar suas condições de produção, prescrição, comercialização, transporte, armazenamento e a ocorrência de eventos adversos advindos de seu uso ${ }^{6}$.

O objetivo deste trabalho foi identificar o estágio de desenvolvimento e o marco regulatório dos medicamentos nanotecnológicos e seus desafios no Brasil, enfatizando a segurança sanitária. Foi realizada análise documental, tendo como fonte de dados os sítios de agências reguladoras nacional (Agência Nacional de Vigilância Sanitária - Anvisa) e internacionais americana (Food and Drug Administration - FDA) e europeia (European Medicines Agency - EMA), bem como do Ministério da Ciência, Tecnologia e Inovação (MCTI), responsável pelo desenvolvimento da nanotecnologia no Brasil. Foi, também, realizada revisão bibliográfica de artigos científicos e legislações encontradas nas bases de dados PubMed, SciELO, Web of Science e Biblioteca Virtual em Saúde (BVS), utilizando as palavras chave: efeito adverso nano ${ }^{\star}$, farmacovigilância, farmacovigilância limitações, farmacovigilância nano* nano\$ risco fármaco, nano\$ risco, nanobiotecnologia, nano* fármacos, nano* segurança, nano ${ }^{\star}$ toxicidade, nanofármacos, nanomedicina, nanotecnologia, nanotoxicologia, pós comercialização nano ${ }^{*}$, vigilância pós comercialização nano $^{*}$, regulamentação nano ${ }^{*}$ e reação adversa nano ${ }^{*}$. A existência de medicamento nanotecnológico registrada na Anvisa foi considerada quando relatado em artigos e/ou quando constatado, no banco de registros da Anvisa, medicamento com a presença de um constituinte nanométrico. A busca do medicamento foi realizada pelo nome comercial, a partir de informação de medicamentos nanotecnológicos registrados no exterior. 


\section{Principais aspectos do desenvolvimento de medicamentos nanotecnológicos}

A nanotecnologia baseia-se em materiais na nanoescala, sendo tão pequenos que podem apresentar propriedades químicas, físicas, biológicas e/ou comportamentais diferentes daquelas existentes nas macroescalas ${ }^{1,2}$. Pode ser aplicada em praticamente todas as áreas da sociedade, sendo necessária para sua elaboração a integração de diversas disciplinas, ou seja, trata-se de uma tecnologia que envolve o conhecimento de forma transdisciplinar e multidisciplinar, respectivamente. Por essas características ela é considerada a via para a resolução de muitos dos problemas da atualidade ${ }^{2,7}$. Seu crescimento pode ser acompanhado pelo aumento do número de artigos e patentes sobre o assunto, já sendo o setor com o maior crescimento na quantidade de depósitos de patentes nas áreas de eletrônica e de medicamentos $^{8,9}$. As patentes são depositadas a partir de seus países de origem ou naqueles com maior mercado para a tecnologia, sendo os de maior desenvolvimento e/ou mercado os Estados Unidos das Américas (EUA), o Japão, a China, a Coreia e a Alemanha ${ }^{8,9}$. Acredita-se que, em 2015 metade do setor farmacêutico mundial dependerá da nanotecnologia ${ }^{10}$. O Brasil, a partir de 2010, aparece em $13^{\circ}$ lugar no ranking de países que tem prioridade para depósito de patentes relacionadas à nanotecnologia e desde 2008 ocupa essa mesma posição entre aqueles com prioridade para depósito de patentes relacionadas à nanobiotecnologia. Isso demonstra a presença brasileira no desenvolvimento de produtos ou processos relacionados à nanotecnologia e à nanobiotecnologia ou o interesse das firmas estrangeiras no mercado nacional ${ }^{8,9,11}$.

A nanociência e a nanotecnologia $(\mathrm{N} \& \mathrm{~N})$, ou seja, o estudo e a aplicação dessa tecnologia, respectivamente, adquiriram maior notoriedade no Brasil a partir dos anos 2000, quando ocorreram as primeiras iniciativas do MCTI de reunir pesquisadores que já atuavam ou que tinham interesse na área ${ }^{12}$. Em 2001, o governo criou quatro redes de pesquisa, que entre 2002 e 2005 envolveram 300 pesquisadores, 77 instituições de ensino e pesquisa, 13 empresas e resultaram na publicação de mais de 1000 artigos científicos e no depósito de mais de 90 patentes ${ }^{12,13}$. Nessa mesma época foi instituído o Programa de "Desenvolvimento da Nanociência e da Nanotecnologia" com o objetivo de desenvolver a competitividade da indústria nacional nesse setor ${ }^{12,13}$. Em 2005, esse programa foi fortalecido com o lançamento da Polí- tica Industrial, Tecnológica e do Comércio Exterior (PITCE) e com a criação da Ação Transversal de Nanotecnologia dos Fundos Setoriais. Suas ações foram inseridas em outro programa mais abrangente: Programa de "Ciência, Tecnologia e Inovação para a Política Industrial, Tecnológica e de Comércio Exterior" com o objetivo de fortalecer a PITCE. Nesse mesmo ano, com o objetivo de desenvolver a N\&N, foi lançado o Programa Nacional de Nanotecnologia $(\mathrm{PNN})^{12}$. A partir de 2008, o investimento em pesquisa e desenvolvimento $(\mathrm{P} \& \mathrm{D})$ da nanotecnologia foi considerado fundamental para elevar a competitividade da economia, uma vez que se espera que contribua de forma significativa para o desenvolvimento industrial do Brasil ${ }^{14,15}$.

Em julho de 2012 foi criado um Comitê Interministerial de Nanotecnologias com o intuito de subsidiar a criação de um Programa de Iniciativas Brasileira em Nanotecnologia, com o objetivo de promover seu desenvolvimento no país, principalmente pela integração das universidades com as indústrias. Acredita-se que a partir de 2013 cerca de R $\$ 40$ milhões por ano serão destinados para esse programa ${ }^{16}$.

O Brasil prioriza o desenvolvimento da nanotecnologia em três mercados específicos: produtos farmacêuticos, químicos e cosméticos, levando em consideração as características de consumo do mercado interno, do desenvolvimento interno do setor químico e farmacêutico e de sua grande biodiversidade ${ }^{17}$.

As indústrias farmacêuticas têm investido na pesquisa e no desenvolvimento de novos medicamentos nanotecnológicos, que possuem sistemas nanoestruturados de liberação de fármacos. Como resultante dessas novas possibilidades de liberação dos fármacos, tem-se como vantagens a redução dos efeitos adversos e da toxicidade do fármaco, proporcionando maior conforto ao paciente e consequentemente, maior adesão ao tratamento. Contudo, esses sistemas também podem apresentar algumas desvantagens, como: 1) toxicidade dos produtos ou dos metabólitos oriundos da sua biodegradação; 2) preço mais elevado, devido ao custo do processo tecnológico, que pode ser compensado pela redução das doses; e, 3 ) possibilidade de permear barreiras biológicas e atingir órgãos nobres como o cérebro ${ }^{10}$.

Os benefícios das N\&N têm sido estudados e bem caracterizados, mas seus riscos ainda permanecem uma questão em aberto ${ }^{18}$. Essa falta de informações sobre os riscos advindos desta nova tecnologia é um dificultador para a tomada das 
decisões governamentais e a regulação da nanotecnologia ${ }^{19}$.

O interesse em estudar os riscos da N\&N tem aumentado e isto pode ser comprovado pelo aumento no número de publicações referentes ao assunto, como pode ser visualizado na Figura $1^{18}$.

Entretanto, estudar e pesquisar sobre os riscos da N\&N não é uma tarefa fácil, já que é extremamente complicado definir quais características serão usadas para avaliação, pois muitas delas são importantes e podem influenciar nos seus efeitos, como: natureza do material, tamanho, forma, fisicoquímica de superfície, solubilidade, carga e outras ${ }^{20}$.

A atual grande questão a ser resolvida em relação à nanotecnologia é como usufruir dos benefícios sem estar exposto aos seus riscos ainda pouco conhecidos.

\section{Medicamentos Nanotecnológicos: regulação necessária?}

Apesar de já existir muitos produtos nanotecnológicos no mercado, inclusive medicamentos, nem todos os países possuem uma regulação específica para esses produtos. Assim, a questão sobre a regulação da nanotecnologia não é se ela ocorrerá e sim quando e de qual forma ${ }^{21}$.

Nos Estados Unidos da América, o FDA é o responsável pela regulação dos medicamentos. Um Grupo de Trabalho foi instituído em 2006, a fim de identificar formas de avaliar os riscos e os benefícios potenciais dos produtos nanotecnológicos que são regulamentados pelo FDA e recomendou a elaboração de um guia para orien-

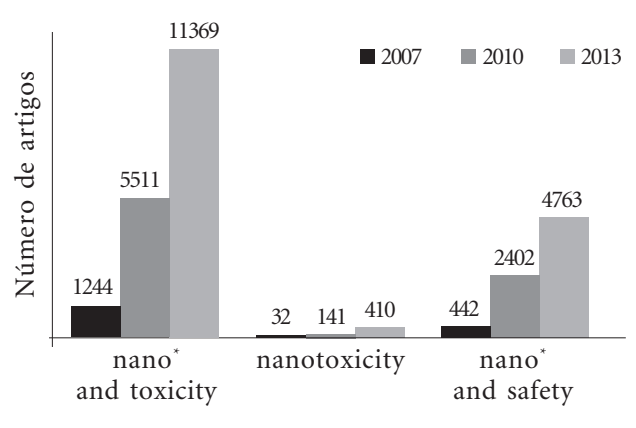

Figura 1. Comparação de publicações referentes ao risco entre os anos de 2007, 2010 e 2013.

Fonte: Chuankrerkkul e Sangsuk ${ }^{18}$ e Web of Science ${ }^{22}$. tar a indústria ${ }^{23}$. A regulação dos produtos nanotecnológicos segue uma política focada nos produtos e com base científica, na qual as análises técnicas e as normas legais são específicas por produto. Além disso, o FDA tem fornecido orientações e assessoria técnica para as indústrias, bem como o controle dos produtos na pós-comercialização ${ }^{24}$. A análise de risco varia de acordo com a classe do produto e leva em conta a segurança, a eficácia e o impacto na Saúde Pública. Essa é a mesma lógica de análise de outros produtos, pois se considera que a avaliação de segurança é suficientemente robusta. Entretanto, o FDA está investindo em um amplo programa de regulação da nanotecnologia a fim de reforçar suas capacidades científicas em avaliar as propriedades dos nanomateriais e o impacto que elas podem ter sobre os produtos ${ }^{24}$. Também tem se buscado obter dados e orquestrar abordagens políticas para garantir a segurança e a eficácia dos produtos nanotecnológicos por meio da Iniciativa Nacional em Nanotecnologia (National Nanotechnology Initiative - NNI), em colaboração com a Casa Branca, com outras agências governamentais norte-americanas e com agências internacionais de regulação ${ }^{23}$.

$\mathrm{Na}$ União Europeia, a regulação dos medicamentos nanotecnológicos ocorre de forma semelhante pela EMA. Diversos documentos foram publicados visando a padronizar os conceitos e orientar as indústrias e pesquisadores a trabalhar conscientemente com a nanotecnologia. Alguns grupos estão se organizando para pesquisar sobre os riscos da nanotecnologia e as formas de avaliá-los. O Comitê Científico sobre Riscos à Saúde Emergentes e Recentemente Identificados (Scientific Committee on Emerging and Newly Identified Health Risks - SCENIHR) identificou a necessidade de uma análise caso a caso na avaliação dos riscos dos nanomateriais ${ }^{25}$. Desde 2006 a EMA tem analisado a aprovação de medicamentos nanotecnológicos e até o momento 20 já foram aprovados ${ }^{25}$. Esses medicamentos são analisados de acordo com a legislação farmacêutica vigente que se baseia na relação benefício/risco, sendo acompanhados após a comercialização ${ }^{26}$.

No Brasil, a responsabilidade de aprovar a entrada no mercado e de regular este medicamentos é do Sistema Nacional de Vigilância Sanitária, em sua esfera federal, a Anvisa. É uma de suas principais e mais antigas áreas de atuação, estando envolvida em todas as etapas do desenvolvimento de medicamentos ${ }^{27}$. As iniciativas legislativas brasileiras iniciaram com o Projeto de Lei no 5.076, de 2005, que procurou regulamen- 
tar a pesquisa e o uso da nanotecnologia no País, criar uma Comissão Técnica Nacional de $\mathrm{Na}$ nossegurança (CTNano), e instituir um Fundo de Desenvolvimento de Nanotecnologia (FDNano), entre outras providências ${ }^{28}$. Esse projeto foi elaborado, em colaboração com dezenas de especialistas, contemplando o desenvolvimento da nanotecnologia em diferentes campos e também sob diferentes aspectos. Recomendava medidas como o monitoramento dos produtos pelos detentores de seu registro após comercialização, a necessidade de identificação no rótulo do produto como nanotecnológico e o incentivo às pesquisas de avaliação de risco; dentre outras medidas que, se implementadas, facilitariam de forma acentuada o trabalho de regulação dos medicamentos nanotecnológicos ${ }^{28}$.

Esse projeto foi avaliado por três comissões diferentes da Câmara Federal, a saber: Comissão de Finanças e Tributação, Comissão de Desenvolvimento Econômico, Indústria e Comércio e Comissão de Ciência e Tecnologia, Comunicação e Informática. Todos os relatores deram pareceres desfavoráveis, seja por incompatibilidade e inadequação orçamentária e financeira, por falta de consenso entre o Executivo e o setor privado sobre como regulamentar a nanotecnologia no país ou por receio de que o controle de risco do projeto pudesse inibir os investimentos na área ${ }^{29-31}$. Os relatores ainda sugeriram que as preocupações com a questão do risco estariam contempladas em outros arcabouços regulatório-legais no país, como a Lei de Biossegurança, Lei no 11.105, de 24 de Março de 2005, sendo que esta se restringe às pesquisas de organismos geneticamente modificados (OGM) $)^{30-32}$. Também cita a Anvisa como responsável pela regulação no campo da saúde, ainda que não possa avaliar os riscos dos medicamentos nanotecnológicos e estabelecer, de forma precisa, sua segurança no momento de seu registro ${ }^{29,33}$.

No caso da rotulagem, os relatores se basearam no Código de Defesa do Consumidor, Lei ${ }^{\circ}$ 8.078/91, e no Decreto no ${ }^{\circ} .680 / 03$ que determina os critérios para rotulagem de alimentos que possuam OGM para inferir que por analogia os produtos nanotecnológicos sejam corretamente identificados ${ }^{30}$. Entretanto, observa-se que infelizmente essa analogia não está funcionando e que atualmente há, no mercado, produtos nanotecnológicos sem identificação.

Em 2010, foi elaborado o Projeto de Lei do Senado $n^{\circ} 131$, que prevê a identificação por meio de símbolos e expressões a constituição nanotecnológica de alimentos, cosméticos, saneantes, medicamentos e correlatos ${ }^{34}$. Em 2013 esse projeto foi rejeitado pela Comissão de Assuntos Sociais (CAS) e pela Comissão de Meio Ambiente, Defesa do Consumidor e Fiscalização e Controle $(\mathrm{CMA})^{35}$.

$\mathrm{O}$ registro desses medicamentos nanotecnológicos, como quaisquer outros novos medicamentos, deve ser feito pela Anvisa e constitui uma etapa fundamental na qual se deve avaliar a eficácia, a segurança e também o ganho terapêuti$\mathrm{co}^{36}$. Entretanto, a falta de regulamentação é um dificultador para a regulação dos medicamentos nanotecnológicos pela Anvisa, que por consequência não tem como saber quais testes devem ser feitos a fim de garantir a segurança desses medicamentos, dificultando seu registro ${ }^{33}$.

Apesar disso, foi possível identificar ao menos 7 medicamentos nanotecnológicos registrados no Brasil até dezembro de 2012 (Quadro 1).

Quatro desses medicamentos, a despeito de serem nanotecnológicos, foram registrados na Anvisa como similares. É considerado medicamento similar, pelo Decreto no 3.961 de 10 de outubro de $2001^{37}$, quando for equivalente ao registrado. O processo de encapsulação/complexação dos fármacos, seja por lipossomas, polímeros ou ciclodextrina, altera a biodisponibilidade desses medicamentos nanotecnológicos quando comparados com o de referência e é justamente essa diferença tida como sua vantagem. Assim, deveriam possuir atenção especial para o seu registro ou, no mínimo, serem registrados como medicamentos novos, já que este conceito, na Resolução RDC MS/ANVISA no 136 de 2003"3, abrange o registro de produtos que possuam alteração na forma farmacêutica, concentração, via de administração e propriedades farmacocinéticas.

Outro agravante é a falta de uma identificação clara na bula desses produtos quanto às suas constituições nanotecnológicas, mesmo naqueles que foram registrados como medicamentos novos. Na bula destinada aos pacientes essa informação não consta em nenhum dos produtos, enquanto que naquela destinada aos profissionais há apenas a referência ao tamanho nanométrico daqueles constituídos por lipossomas. As embalagens também não indicam claramente tratar-se de um medicamento nanotecnológico. Ressalte-se, portanto, que devido à dificuldade de encontrar informações sobre a presença de constituintes nanométricos e a não referência a esta característica quando do registro do medicamento, limitou-se a busca, podendo haver outros registros não identificados. Sant'Anna et al. ${ }^{39}$ identificaram, também outros medicamentos 


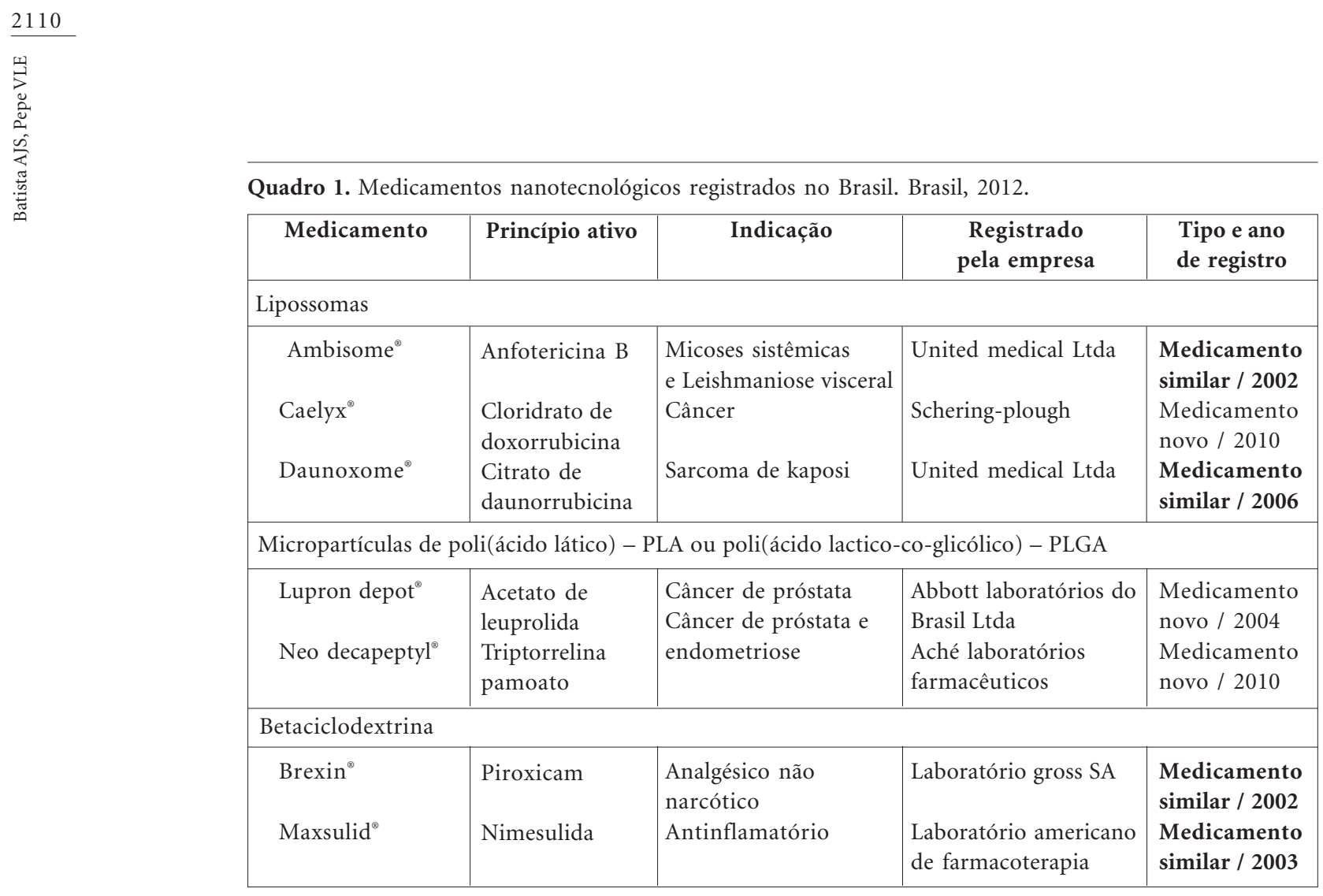

nanotecnológicos comercializados no Brasil: Doxil $^{\oplus}$ (doxorubicin), Amphocil ${ }^{\oplus}$ e Abelcet $^{\oplus}$ (anfotericina B), Rapamune ${ }^{\circledast}$ (sirolimus), Emend ${ }^{\circledast}$ (aprepitant,MK869), TriCor ${ }^{\varpi}$ (fenofibrate), Megace $\mathrm{ES}^{\circledast}$ (megaestrol, acetato), Abraxane ${ }^{\circledast}$ (paclitaxel), Elestrin ${ }^{\circledast}$ (estradiol).

Como não há uma informação clara que esses medicamentos são nanotecnológicos nenhum monitoramento específico tem sido feito com seus usuários, não fornecendo valiosos dados para a avaliação da segurança desses medicamentos. Todos esses fatos evidenciam a necessidade de uma correta regulação dos medicamentos nanotecnológicos, para que estes possam entrar no mercado corretamente, de forma que todos os seus benefícios sejam aproveitados e seus riscos minimizados.

A VISA enfrenta desafios em relação às novas tecnologias, já que é sua competência regular boa parte daquelas em saúde e, inclusive, monitorar os eventos adversos (e riscos) dos recursos tecnológicos, produtos e serviços utilizados pelo sistema de saúde. No caso de medicamentos nanotecnológicos, há, além das dificuldades usuais, a ausência, a insuficiência ou a incerteza de informações científicas. Nestes casos, as decisões regulatórias devem levar em conta o princípio da precaução, o qual propõe o uso controlado dos medicamentos até que novos resultados sobre a avaliação do risco sejam obtidos, de forma a proteger o ambiente, o consumidor e também o trabalhador ${ }^{40,41}$. No contexto do princípio da precaução, o gerenciamento de risco da nanotecnologia deve dar origem a estudos que façam uma análise do possível custo/benefício dos produtos nanotecnológicos que entrarão no mercado, permitindo que produtos com um risco aceitável entrem e tenham seus benefícios aproveitados, já que nem sempre é possível reduzir o risco a zero. Essa análise e a regulação de entrada e comercialização devem estar em constante atualização de acordo com novos dados científicos que surjam e devem ser realizadas de forma específica por categoria do produto. Para isso, é necessário desenvolver e incorporar métodos científicos de avaliação e gerenciamento de riscos, assim como técnicas que permitam a avaliação da eficácia, da segurança e da relação custo/benefício ${ }^{19}$. A VISA deve contar com laboratórios modernos e bem equipados, assim como com profissionais qualificados para fornecer resultados fidedignos na avaliação da qualidade de produtos e nas caracterizações de riscos e agravos para a saúde dos usuários ${ }^{42}$.

Em alguns países, as atividades de pesquisa estão vinculadas direta ou indiretamente ao Ministério da Saúde facilitando a articulação entre a produção do conhecimento científico, as decisões 
políticas e as atividades regulatórias no campo da saúde $^{19}$. No Brasil, as pesquisas em nanotecnologia estão vinculadas ao MCTI, mas cabe à Anvisa o papel de avaliar os benefícios obtidos com o desenvolvimento científico e, ao mesmo tempo, agir de modo a garantir a precaução no domínio da saúde pública, permitindo assim que apenas medicamentos seguros entrem no mercado ${ }^{43}$.

$\mathrm{O}$ fato do conceito brasileiro de biossegurança se restringir a pesquisas de organismos geneticamente modificados (OGM), de acordo com a Lei Federal no 11.105, de 24 de março de 2005, também dificulta as ações de avaliação de risco das pesquisas em $\mathrm{N} \& \mathrm{~N}^{32}$.

Outra ação da VISA e, portanto, desafio, é a ação de comunicação científica e técnica assim como a educação sanitária da população. Para isso, esta deve reunir informações científicas e apreender a percepção popular a fim de transmitir esses conhecimentos de forma compreensível para o público. Desta maneira pode contribuir para a construção de uma consciência sanitária, permitindo tanto a escolha quanto a aceitação de risco mais qualificada e dando meios de defesa contra inúmeros riscos e práticas nocivas do sistema produtivo ${ }^{42}$. Entretanto, essa ação ainda parece ser tímida, especialmente em relação à N\&N.

Há ainda o desconhecimento ou a baixa percepção do risco da nanotecnologia no meio acadêmico e nas indústrias farmacêuticas. Schulte e Salamanca-Buentello ${ }^{40}$ destacaram como questões éticas para a saúde do profissional que trabalha com nanotecnologia a identificação e comunicação dos riscos por cientistas, autoridades e empregadores e sua aceitação por parte desses trabalhadores. Entretanto, ainda existem profissionais da área farmacêutica e biomédica que manipulam produtos nanotecnológicos apesar de não possuírem o conhecimento, a infraestrutura e as roupas adequadas para tal. $\mathrm{O}$ fato de a população não ter ainda informações suficientes é evidenciada pela ausência de organizações sociais nos processos decisivos para debater ou questionar as decisões governamentais, a fim de que as necessidades sociais sejam atendidas e não só os interesses econômicos 5 .

\section{Conclusão}

Os desafios que a Anvisa terá para regular os medicamentos nanotecnológicos não difere mui- to daqueles que já enfrenta atualmente. Entretanto, no caso da nanotecnologia eles serão acentuados, pois os testes exigirão pessoas mais especializadas, equipamentos mais sofisticados e as avaliações de qualidade deverão abranger novos parâmetros como a caracterização do tamanho de partícula. Além disso, a falta de pesquisas e incentivos na caracterização dos riscos da nanotecnologia dificulta a avaliação e o gerenciamento pela Anvisa dos riscos dos medicamentos nanotecnológicos.

Tendo em vista essa falta de comprovação científica do risco, sugere-se que a regulação adotada deva ser baseada no princípio da precaução e que a avaliação de risco seja feita caso a caso, seguindo os modelos implantados pelo FDA e pela EMA. As principais ações que devem ser contempladas na regulação dos medicamentos nanotecnológicos são:

- registro como medicamento novo;

. acompanhamento estreito do Programa de Farmacovigilância da indústria produtora;

. efetivação de programas de investigação dos efeitos toxicológicos dos medicamentos nanotecnológicos;

- comunicação à população e aos profissionais de saúde sobre a nanotecnologia e (a falta de informação sobre) seus efeitos, tanto pela inserção de informações no sítio da Anvisa como pela utilização de outras estratégias;

- obrigatoriedade de informar claramente na embalagem e na bula que se trata de um produto nanotecnológico;

- programa de monitoramento específico do desenvolvimento clínico e do uso do medicamento pelo produtor.

Conclui-se que o principal desafio para a regulação da nanotecnologia consiste na conscientização de todos os atores envolvidos - pesquisadores, trabalhadores, reguladores, produtores e consumidores. Em face às insuficientes informações científicas sobre a segurança desses produtos, sugere-se que a regulação considere o princípio da precaução, sendo o registro feito como medicamento novo, a população e os profissionais de saúde informados e o uso monitorado de forma a que novos resultados sobre a avaliação de seu risco sejam obtidos e novos conhecimentos possam subsidiar a avaliação da decisão regulatória, de forma a ratificá-la se os benefícios se mostrarem superiores aos riscos ou, ao contrário, retificá-la em face à descoberta de graves eventos adversos oriundos de seu uso. 


\section{Colaboradores}

AJ Sousa-Batista e VLE Pepe participaram na concepção do trabalho, na pesquisa bibliográfica e na redação do artigo.

\section{Referências}

1. Melo CP, Pimenta M. Nanociências e nanotecnologia. Parcerias estratégicas 2004; 18:9-21.

2. Medeiros ES, Paterno LG, Mattoso LHC. Nanotecnologia. In: Durán N, Mattoso LHC, Morais PC, organizadores. Nanotecnologia Introdução, preparação e caracterização de nanomateriais e exemplos de aplicação. São Paulo: Artliber; 2006. p. 13-29.

3. Agência Brasileira de Desenvolvimento Industrial (ABDI). Cartilha sobre nanotecnologia. 2010. [acessado $2010 \mathrm{dez}$ 13]. Disponível em: http://www.abdi. com.br/?q=system/files/Cartilha.pdf.

4. Agência Brasileira de Desenvolvimento Industrial (ABDI). Nanotecnologia. Estudo Prospectivo Nanotecnologia: 2008-2025. (Sem Data). [acessado 2010 dez 14] Disponível em: http://www.abdi.com.br/ $? \mathrm{q}=$ system/files/Relatorio_INI_ Nanotecnologia_ Estudo+Prospectivo.pdf.

5. Foladori G, Figueroa S, Edgard Z, Invernizz i N. Características distintivas del desarrollo de las nanotecnologias en América Latina. Sociologias 2012; 14(30):330-363.

6. Almeida Filho NA. O conceito de saúde e a Vigilância Sanitária: notas para a compreensão de um conjunto organizado de práticas de saúde. In: Costa AE, organizadora. Vigilância Sanitária desvendando o enigma. Salvador: EDUFBA; 2008. p. 19-43.

7. Centro Ecológico. Nanotecnologia: A manipulação do invisível. Novas Tecnologias 2009; 44p.

8. Nunes J. Pedidos de Patente sobre Nanotecnologia publicados no $1^{\circ}$ semestre de 2010. INPI/DART/ CEDIN/DIESPRO. Alerta Tecnológico no 29; 2010. [acessado 2010 dez 14]. Disponível em: www.inpi. gov.br/menu-esquerdo/...em.../alerta_nano_jan _jun_2010.pdf.

9. Nunes J, Rohem-Santos P. Pedidos de Patente sobre Nanotecnologia publicados no $2^{\circ}$ semestre de 2009. INPI/DART/CEDIN/DIESPRO. Alerta Tecnológico $n^{\circ}$ 20; 2010. [acessado 14 dez. 2010]. Disponível em http://www.inpi.gov.br/menu-esquerdo/informacao/ alerta_nano_20.pdf.

10. Rossi-Bergman, B. A nanotecnologia: da saúde para além do determinismo tecnológico. Cienc Cult 2008; 60(2):54-57.

11. Nunes J, Rohem-Santos P. Pedidos de Patente sobre Nanobiotecnologia publicados no $2^{\circ}$ semestre de 2008. INPI/DART/CEDIN/DIESPRO. Alerta Tecnológico $n^{o} 18 ; 2010$. [acessado 14 dez. 2010]. Disponível em: http://www.inpi.gov.br/menu-esquerdo/ informacao/alerta_nanobio_n18.pdf.

12. Fernandes MFM, Filgueiras CAL. Um panorama da nanotecnologia no Brasil (e seus macro-desafios). Quim. Nova 2008; 31(8):2205-2213.

13. Brasil. Ministério da Ciência e Tecnologia (MCT). Nanotecnologia. Investimentos, Resultados e Demandas. 2006. [acessado $2010 \mathrm{dez}$ 12]. Disponível em: http://www.mct.gov.br/upd_blob/0019/19536.pdf. 
14. Brasil. Ministério do Planejamento, Orçamento e Gestão (MPOG), Secretaria de Planejamento e Investimentos Estratégicos. Plano plurianual 20082011: projeto de lei. Brasília. MP 2007; 2: 540 p. [acessado $2010 \mathrm{dez}$ 14]. Disponível em: http://www. sigplan.gov.br/download/avisos/001 mensagem presidencial_internet.pdf.

15. Brasil. Ministério do Planejamento, Orçamento e Gestão (MPOG), Secretaria de Planejamento e Investimentos Estratégicos. Plano plurianual 20122015: projeto de lei. Brasília. MP 2011; 278 p. [acessado 2012 jul 19]. Disponível em: http://www. planejamento.gov.br/secretarias/upload/Arquivos/ spi/PPA/2012/mensagem_presidencial_ppa.pdf

16. Martins C. MCTI prepara lançamento do programa Iniciativa Brasileira em Nanotecnologia. Boletim Eletrônico de Notícias da Sociedade Brasileira de Química de 19 de julho de 2012 - No 1034. [acessado 2012 jul 19]. Disponível em: http://boletim.sbq. org.br/noticias/n464.php

17. Agência Brasileira de Desenvolvimento Industria (ABDI). Panorama Nanotecnologia. Série Cadernos da Indústria ABDI. Volume XIX. Brasília; 2010. [acessado 2010 dez 14]. Disponível em: http://www.abdi .com.br/Estudo/Panorama\%20de\%20Nanotecnologia .pdf

18. Chuankrerkkul N, Sangsuk S. Current Status of Nanotechnology Consumer Products and Nano-Safety Issues. JOM 2008, 18(1):75-79.

19. Barreto ML. O conhecimento científico e tecnológico como evidência para políticas e atividades regulatórias em saúde. In: Costa AE, organizadora. Vigilância Sanitária desvendando o enigma. Salvador: EDUFBA; 2008. p. 91-106.

20. Williams RA, Kulinowski KM, White R, Louis G. Risk Characterization for Nanotechnology. Risk Anal 2010; 30(11):1671-1679.

21. Marchant GE, Sylvester DJ, Abbott KW. What Does the History of Technology Regulation Teach Us about Nano Oversight? J Law Med Ethics 2009; 37(4):724-731.

22. Web of Science. [acessado $2010 \mathrm{dez} 15$ e $2013 \mathrm{mar}$ 27]. Disponível em: http://apps.webofknowledge. com/UA_GeneralSearch_input.do?product=UA\& search $\_$mode $=$GeneralSearch \&SID $=$R2SR2hVWJrfIwa JjMJJ\&preferencesSaved =

23. Food and Drug Administration (FDA). FDA Continues Dialogue on 'Nano' Regulation. FDA Consumer Health Information / U. S. Food and Drug Administration. 2012. [acessado 2012 jul 20]. Disponível em: http://www.fda.gov/downloads/For Consumers/ConsumerUpdates/UCM258691.pdf
24. Food and Drug Administration (FDA). Fact Sheet: Nanotechnology. Nanotechnology Overview. 2012. [acessado 2012 jul 20]. Disponível em: http:// www. fda.gov/Food/GuidanceComplianceRegulatory Information/GuidanceDocuments/ucm300914.htm12k-2012-04-20

25. European Union (UE). Commission Staff Working Paper. Types and uses of nanomaterials, including safety aspects. Accompanying the Communication from the Commission to the European Parliament, the Council and the European Economic and Social Committee on the Second Regulatory Review on $\mathrm{Na}$ nomaterials. Brussels: EU; 2012.

26. European Union (UE). Commission Staff Working Paper. Accompanying document to the Communication From The Commission To The European Parliament, The Council And The European Economic And Social Committee Regulatory Aspects Of Nanomaterials. Summary of legislation in relation to health, safety and environment aspects of nanomaterials, regulatory research needs and related measures. Brussels: EU; 2008

27. Pepe VLE, Reis LGC, Noronha MF, Schramm JM. Avaliação em Saúde e Vigilância Sanitária: conceitos, estratégias e metodologias. In: De Seta $\mathrm{MH}$ Pepe VLE, Oliveira GO, organizadores. Gestão e Vigilância Sanitária: modos atuais do pensar e fazer. Rio de Janeiro: Fiocruz; 2006. p. 251-276.

28. Brasil. Projeto de Lei No 5.076, de 2005. Deputado Edson Duarte. Brasília, DF. 2005. [acessado 2011 mar 25]. Disponível em: http://www.camara.gov.br/ sileg/integras/297210.pdf.

29. Brasil. Câmara dos Deputados Comissão de Financas e Tributação. Relatório do Projeto de Lei N $N^{\circ}$ 5.076, de 2005. Relator Deputado Duarte Nogueira. Brasília, DF. 2008a. [acessado 2011 mar 25]. Disponível em: http://www.camara.gov.br/sileg/integras/602144. pdf

30. Brasil. Comissão de Ciência e Tecnologia, Comunicação e Informática. Relatório do Projeto de Lei $N^{o}$ 5.076, de 2005. Relatora Deputada Luiza Erundina. Brasília, DF. 2008b. [acessado 2011 mar 25]. Disponível em: http://www.camara.gov.br/sileg/integras/ 567046.pdf

31. Brasil. Comissão de Desenvolvimento Econômico, Indústria e Comércio. Relatório do Projeto de Lei $N^{o}$ 5.076, de 2005. Relator Deputado Léo Alcântara. Brasília, DF. 2005. [acessado 2011 mar 25]. Disponível em: http://www.camara.gov.br/sileg/integras/ 315967.pdf

32. Silva RP. A nova lei brasileira de biossegurança e o instituto da responsabilidade civil. Biblioteca juridical virtual del instituto de investigaciones jurídicas de la UNAM. 2007. [acessado $2010 \mathrm{dez} 26$ ]. Disponível em: http://www.juridicas.unam.mx. 
33. Caballero NED. Debate: Propaganda de produtos com nanopartículas. In: Emerick ME, Montenegro KBM, Degrave W, organizadores. Novas Tecnologias na Genética Humana: Avanços e Impactos para a Saúde. Rio de Janeiro: Fiocruz; 2007.

34. Brasil. Senado Federal. Projeto de Lei do Senado $N^{o}$ 131, de 2010. Secretaria-Geral da Mesa. [acessado 2012 ago 14]. Disponível em: http://www.senado. gov.br/atividade/materia/detalhes.asp?p_cod_ mate $=96840$.

35. Brasil. Senado Federal. Comissão de Assuntos Sociais. Parecer sobre o Projeto de Lei do Senado No 131, de 2010. Relator Senador Cícero Lucena. 2013. [acessado 2013 mar 27]. Disponível em: http://www. senado.gov.br/atividade/materia/getPDF.asp? $\mathrm{t}=$ $124324 \& \operatorname{tp}=1$.

36. Gava CM, Bermudez JAZ, Pepe VLE, Reis ALA. Novos medicamentos registrados no Brasil:podem ser considerados como avanço terapêutico? Cien Saude Colet 2010; 15(3):3403-3412.

37. Brasil. Decreto no 3.961, de 10 de outubro de 2001 Altera o Decreto no 79.094, de 5 de janeiro de 1977 que regulamenta a Lei no 6.360 , de 23 de setembro de 1976. Diário Oficial da União 2001; 11 out.

38. Brasil. Ministério da Saúde (MS). Agência Nacional de Vigilância Sanitária (Anvisa). Resolução RDC $n^{\circ} 136$, de 29 de maio de 2003. Dispõe sobre o registro de medicamento novo. Diário Oficial da União 2003; 12 ago.

39. Sant'Anna LS, Ferreira AP, Maria Alencar MSM. Patents, drug delivery and public health protection: health risk management for nanopharmaceuticals. J Technol Manag Innov 2013, 8(2):107-118.

40. Schulte PA, Salamanca-Buentello F. Ethical and scientific issues of nanotechnology in the workplace. Cien Saude Colet 2007, 12(5):1319-1332.

41. Von Hohendorff R, Engelmann W, Oshirov M. As nanotecnologias no meio ambiente do trabalho: a precaução para equacionar os riscos do trabalhador. Cadernos Ibero-Americanos de Direito Sanitário 2013, 2(2):668-683.

42. Costa EA, Rozenfeld S. Constituição da Vigilância Sanitária no Brasil. In: Rozenfeld S, organizador. Fundamentos da Vigilância Sanitária. Rio de Janeiro: Fiocruz; 2009. p. 15-40

43. Dallari SG. Vigilância Sanitária: Responsabilidade pública na proteção e promoção da saúde. In: Costa AE, organizador. Vigilância Sanitária desvendando o enigma. Salvador: EDUFBA; 2008. p. 45-52. 